\title{
Thaumatelsonella kingelepha, eine neue Gattung und Art aus der Antarktis (Crustacea: Amphipoda: Gammaridea: Stenothoidae)
}

\author{
M. Rauschert ${ }^{1} \&$ H. G. Andres ${ }^{2, *}$ \\ ${ }^{1}$ Forschungsstelle für Wirbeltierforschung (im Tierpark Berlin); Am Tierpark 125, \\ D-O-1136 Berlin, Bundesrepublik Deutschland \\ ${ }^{2}$ Biologische Anstalt Helgoland; Notkestr. 31, D-W-2000 Hamburg 52, \\ Bundesrepublik Deutschland \\ und \\ Zoologisches Institut und Zoologisches Museum der Universität Hamburg; \\ Martin-Luther-King-Platz 3, D-W-2000 Hamburg 13, Bundesrepublik Deutschland * *
}

\begin{abstract}
Thaumatelsonella kingelepha, a new genus and species from Antarctica (Crustacea: Amphipoda: Gammaridea: Stenothoidae). A taxonomic study on stenothoid amphipods from the area of South Shetland Islands (Antarctica) led to the description of Thaumatelsonella kingelepha gen. and sp. nov. The systematic position of Pseudothaumatelson cyproides Nicholls, 1938 is discussed, as the redescription by Bellan-Santini \& Ledoyer (1986) based on the first recovery of that species included differences from the diagnosis of the genus.
\end{abstract}

\section{EINLEITUNG}

Bei der taxonomischen Bearbeitung antarktischen Amphipoden-Materials wurden Stenothoiden gefunden, die wegen Übereinstimmung in wesentlichen Merkmalen (schlanke, rechteckige Basen an Pereiopoden V bis VII, deutlich verlängertes Pereionsegment IV, gut entwickelte Innenlade an Maxille II; vgl. Barnard, 1972a) enge verwandtschaftliche Beziehungen zur Thaumatelsoninen-Gruppe aufweisen. Innerhalb dieser Sektion zeigen sie eine auffällige habituelle Ähnlichkeit mit der von Schellenberg (1931) beschriebenen Gattung und Art Pseudothaumatelson patagonicum. Die anfänglich vermutete Identität mit dieser Spezies fand jedoch keine Bestätigung beim Vergleich mit dem Typusmaterial. Dieser Vergleich ermöglichte Berichtigungen und Ergänzungen der Originalbeschreibung und führte schließlich zur Errichtung einer neuen Gattung und Art für das vorliegende Material; darüber hinaus ließ er die von Bellan-Santini \& Ledoyer (1986) vorgenommene Zuordnung ihres Materials von Marion Island in die Gattung und Art Pseudothaumatelson cyproides Nicholls, 1938 zweifelhaft erscheinen, da sich wesentliche Unterschiede zur Gattungsdiagnose ergaben.

\footnotetext{
- Mitglied der Taxonomischen Arbeitsgruppe an der Biologischen Anstalt Helgoland

* Korrespondenz-Anschrift
} 
Die Sektion Thaumatelsoninae soll sensu Barnard (1972a, b) nicht als phylogenetische Einheit, sondern als eine durch morphologische Kennzeichen diagnostizierbare Gruppe verstanden werden. Intermediäre Merkmalausbildung läßt die Eigenständigkeit der Sektionen Thaumatelsoninae und Stenothoinae bezweifeln. So ist das im Konzept der Thaumatelsoninen hervorgehobene Merkmal "verlängertes Pereionsegment IV" nicht ausschließlich auf diese Gruppe beschränkt. Möglicherweise bleibt als einziges diagnostisches Merkmal für die Thaumatelsoninen die Ausbildung schlanker, linearer (rechteckiger) Basen an Pereiopod V-VI.

\section{MATERIAL}

Das der Bearbeitung zugrunde liegende Material wurde im Gebiet der Antarktischen Halbinsel (South Shetland Islands) eingebracht. Es entstammt (a) Dredschfängen (Maschenweite 0,3 mm), die im Rahmen der Bonitierung der Sublitoralfauna von King George Island von einem der Autoren (M. Rauschert) während mehrjähriger Aufenthalte (1981-82; 1984-86) durchgeführt wurden, und (b) Benthosaufsammlungen, die während der "Walther-Herwig"-Reise 68, 1984/85 im Gebiet der Elephant Island gewonnen wurden (Gerät: Greifer nach van Veen, $0,1 \mathrm{~m}^{2}$ ). Im ganzen lagen 6 Exemplare der als neu $\mathrm{zu}$ beschreibenden Art vor.

Zum Vergleich konnte das Typmaterial von Pseudothaumatelson patagonicum herangezogen werden.

Das Material wird im Zoologischen Museum Berlin (ZMB), Humboldt Universität, und im Zoologischen Museum Hamburg (ZMH) aufbewahrt.

Folgende Abkürzungen finden Verwendung: A: Antenne; AL: Außenlade; Cox: Coxalplatte; Gnp: Gnathopod; IL: Innenlade; Md: Mandibel; P: Pereiopod; Up: Uropod.

Längenangaben beziehen sich auf die Strecke zwischen den Gliedgrenzen, wenn nicht anders angegeben.

\section{TAXONOMIE}

Die Kombination charakteristischer Habitus-Merkmale der vorliegenden Individuen - nasenartiger Dorsodistalfortsatz an Schaftglied 1 der Antenne I, caudad gerichtete Verlängerung am Urosomsegment I, horizontal angeordnetes Telson - liegt innerhalb der Thaumatelsoninen-Gruppe nicht nur in der Gattung Pseudothaumatelson Schellenberg, 1931 vor, sondern ist auch bei Parathaumatelson Gurjanova, 1938 und Chucullba Barnard, 1974 zu finden. Den Darstellungen von Barnard (1972a, b, 1974) folgend, sind Parathaumatelson und Chucullba vor allem wegen folgender Merkmale auszugrenzen: Gnathopod I und II von gleicher Gestalt, Mandibel ohne Palpus, Urosomsegmente II und III basal verschmolzen; darüber hinaus sind Außen- und Innenladen von Maxille II reduziert; die Uropoden sind in Lateralansicht gestaffelt angeordnet (Reichweite von Up I nach Up III abnehmend). Bei Chucullba sind zusätzlich die Innenäste von Uropod I und II stark reduziert, der Ast von Uropod III ist nur eingliedrig.

Bereits Barnard (1974) hat darauf verwiesen, daß die Bedeutung des Merkmals "nasenartiger Fortsatz an Antenne I" als Gattungskriterium wesentlich durch die Tatsache gemindert wird, daß bei einer Art der Gattung Chucullba - C. warea Barnard, 1974 dieser Fortsatz fehlt. Unter diesem Aspekt war bei der Determination noch die Gattung Raumahara Barnard, 1972 zu berücksichtigen. Dieses Genus ist ebenfalls durch ein verlängertes Pereionsegment IV, einen Fortsatz an Urosomsegment I und ein horizontal 
angeordnetes Telson gekennzeichnet, es ist aber deutlich gegen das neue antarktische Material durch den chelaten Gnathopod II unterschieden.

Übereinstimmung mit der im Antiboreal verbreiteten Gattung Probolisca Gurjanova, 1938 ist für folgende Merkmale aufzuzeigen: Bàsen der Pereiopoden V-VII schlank, rechteckig; Mandibularpalpus 3gliedrig; Maxillularpalpus 2gliedrig, Pereionsegment IV verlängert. Die Vertreter der Gattung Probolisca haben jedoch mit Ausnahme von $P$. nasutigenes (Stebbing, 1888) keine Körperfortsätze; bei nasutigenes trägt lediglich Schaftglied 1 der Antenne I distolateral einen spitz auslaufenden Fortsatz.

Einen 3gliedrigen Mandibelpalpus sowie einen 2gliedrigen Maxillularpalpus und Fortsätze an Urosomsegment I und Antenne I weisen innerhalb der Thaumatelsoninen auch Thaumatelson Walker, 1906 und Antatelson Barnard, 1972 auf; diese beiden Gattungen sind aber durch ein vertikal angeordnetes, großflächigeres Telson sowie Verwachsungen von Urosomsegmenten ausgezeichnet.

Die wegen der erheblichen Ähnlichkeit zuerst vermutete Identität mit Pseudothaumatelson patagonicum ließ sich nicht bestätigen, da die vorliegenden Individuen statt eines eingliedrigen Mandibularpalpus einen 3gliedrigen besitzen. Die Wertung des Merkmals „Anzahl der Glieder am Mandibularpalpus“ für die Taxonomie der Stenothoiden und die allgemein übliche diagnostische Handhabung dieses Kriteriums innerhalb der Familie läßt die Erstellung einer eigenen Gattung für diese neue Art als gerechtfertigt erscheinen. Darüber hinaus ist auffällig, daß das Urosom bei Pseudothaumatelson einer Größenreduktion unterliegt.

Nach Barnard $(1972 a, b)$ umfaßt die Gattung Pseudothaumatelson außer der Typusart $P$. patagonicum wahrscheinlich auch die von Nicholls (1938) beschriebene Spezies $P$. cyproides. Eine eindeutige taxonomische Zuordnung von cyproides blieb fraglich, weil in der Originalbeschreibung detaillierte Angaben zu Mandibel, Maxillen, Uropod III und Telson fehlten. Doch auch nach ausführlicher Ergänzung' in Beschreibung und Zeichnung an Hand eines vermeintlichen ersten Wiederfundes für cyproides durch BellanSantini \& Ledoyer (1986: 425 ff., Fig. 29), muß die taxonomische Einordnung von cyproides offenbleiben; in ihrer Darstellung existieren erhebliche morphologische Abweichungen an der Maxille I gegenüber der Gattung Pseudothaumatelson und die Identität zwischen Typus und Wiederfund-Material wird nicht belegt.

Nach der Beschreibung bei Bellan-Santini \& Ledoyer ist cyproides die einzige Art innerhalb der Thaumatelsoninen mit einem eingliedrigen Palpus an Maxille I. Auch die Innenlade der Maxille I ist offensichtlich abweichend gestaltet und bewehrt: sie ist nur wenig kürzer als die Außenlade, distal verjüngt und apikal mit 3 Borsten besetzt. Im Gegensatz dazu haben die übrigen Thaumatelsoninen eine kurze, zungenförmige und mit höchstens 1 distomarginalen Borste versehene Innenlade. Außerdem ist nach der Zeichnung (:426, Fig. 29) die Außenlade der Maxille I apikal nur mit 4 statt 6 Stacheln besetzt. Ansonsten entspricht der Habitus dem von Pseudothaumatelson (Fortsätze an Antenne I sowie Urosomsegment $I_{i}$ verlängertes Pereionsegment IV; horizontale Lagerung des Telsons; Uropoden I und III überragen Up II).

Die angeführten Unterschiede gegenüber der Gattung Pseudothaumatelson müßten konsequenterweise zu einer generischen Trennung zumindest der Individuen, die der Wiederbeschreibung von Bellan-Santini \& Ledoyer zugrunde liegen, führen. 


\section{Pseudothaumatelson Schellenberg, 1931}

D i a g n os e: Gnathopod I und II subchelat, differierend in Form und Größe (Gnp II größer); Palma schräg, an Gnp II kürzer als Propodus-Hinterrand; Carpus jeweils kürzer als Propodus. Mandibel-Palpus eingliedrig. Maxille I: Palpus 2gliedrig; Innenlade kurz, breit-zungenförmig, distal mit 1 Borste. Antenne I mit dorsodistalem Fortsatz an Schaftglied 1. Basen der Pereiopoden V bis VII schlank, rechtwinklig. Pereionsegment IV stark verlängert. Urosomsegmente I bis III nicht verwachsen. Urosomsegment I mit dorsodistalem, weit überragenden und gerundeten Fortsatz. Anordnung der Uropoden in Lateralansicht: Up I und Up III überragen Up II. Telson horizontal gelagert; Lateralränder aufgewölbt.

Typu sart: Pseudothaumatelson patagonicum Schellenberg, 1931: 110, Fig. 60.

Be zi ehungen: Wie Pseudothaumatelson sind auch Parathaumatelson und Chucullba äußerlich durch einen dorsodistalen Fortsatz an Schaftglied 1 der Antenne I sowie an Urosomsegment I und ein horizontal ausgerichtetes Telson charakterisiert. Gegenüber Parathaumatelson und Chucullba ist Pseudothaumatelson u. a. durch freie Urosomsegmente sowie ungleiche Ausgestaltung der Gnathopoden abgegrenzt und darüber hinaus noch durch einen eingliedrigen Mandibel-Palpus gekennzeichnet. Gegenüber der neuen Gattung Thaumatelsonella ist Pseudothaumatelson durch den eingliedrigen Mandibularpalpus deutlich unterschieden, außerdem weist in dieser Gattung das Urosom eine Größenreduktion auf. - Das Erscheinungsbild eines "rudimentarily carpochelate gnathopod 2" (Barnard, 1972a) wird wesentlich durch den schlank ausgebildeten Carpalfortsatz und an den proximal konkav verlaufenden Propodus-Hinterrand verursacht.

Arten: Pseudothaumatelson patagonicum Schellenberg, 1931; ? P. cyproides Nicholls, 1938.

Das Verbreitungsgebiet der Gattung ist die Antarktis (Adélie Coast, Commonwealth Bay) und Magellan Region (Falkland-Inseln). Die bisher bekannte Tiefenverbreitung umfaßt eine Spanne von 22 bis $197 \mathrm{~m}$.

\section{Pseudothaumatelson patagonicum Schellenberg, 1931}

(Abb. 1, A-I)

A n merkungen: Nachstehende Abweichungen sind am Typusmaterial gegenüber der Originalbeschreibung gefunden worden. Gnathopod I: Carpus ist nicht so lang wie Propodus, sondern erreicht nur $4 / 5$ von dessen Länge (Vorderrand); Palmarecke nicht durch 2 Paar Stacheln markiert, sondern durch 3 Paare. Ergänzende Beschreibung zur Palma: Palma-Rand verläuft im ganzen konvex und ist gesägt. Gnathopod II: Form des Palma-Randes erscheint eher gewellt als "fein gekörnt". Pereiopoden VI und VII: Vorderränder der Basen verlaufen leicht konvex, Hinterränder leicht konkav; dadurch sind die schlanken Basen schwach geschwungen; sie sind im ganzen wenig verbreitert. Auch Coxalplatte VI hat wie Cox VII ventrodistal einen kleinen Zahn. Grenzen zwischen Urosomsegmenten I und II sowie II und III verlaufen nicht so steil wie bei Schellenberg (1931: 111, Abb. 60a); die Größe des Urosoms erscheint dadurch reduziert. 


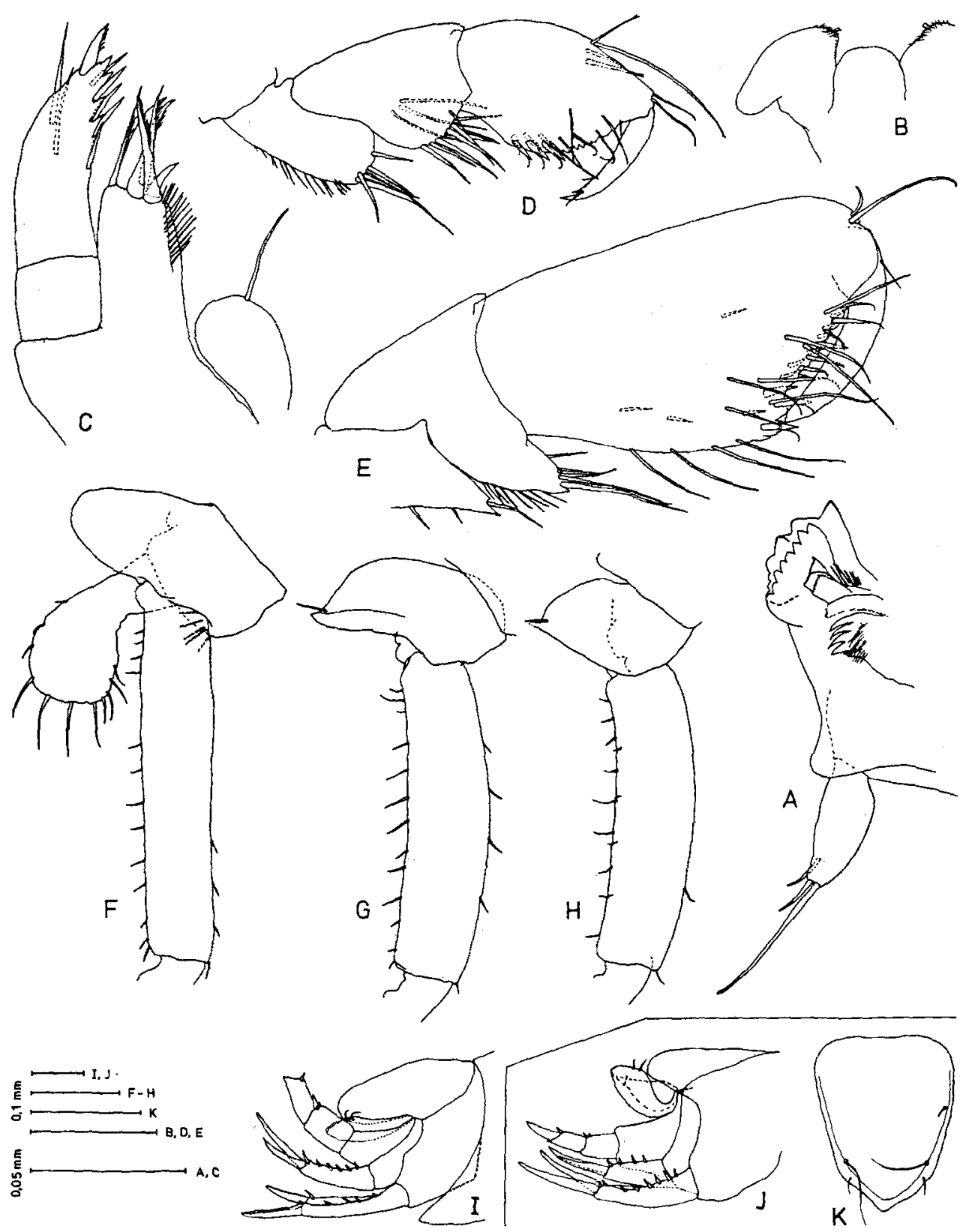

Abb. 1. A-I: Pseudothaumatelson patagonicum Schellenberg, 1931; Holotypus, $q$. $\mathrm{J}$ und $\mathrm{K}$ : Thaumatelsonella kingelepha gen. und $\mathrm{sp} . \mathrm{n}_{\mathrm{i}}$ Holotypus, $q$.

A: Mandibel. B: Unterlippe. C: Maxille I. D: Gnathopod I. E: Gnathopod II. F-H: Pereiopoden V-VII (Coxalplatte und Basis). I und J: Urosom (lateral). K: Telson 


\section{Thaumatelsonella gen. nov.}

Diagn ose: Gnathopod I und II subchelat, unterschiedlich in Größe und Form, Carpus jeweils kürzer als Propodus; Palma schräg, an Gnathopod II kürzer als PropodusHinterrand. Mandibel mit 3gliedrigem Palpus. Palpus von Maxille I 2gliedrig. Schaftglied 1 der Antenne I mit dorsodistalem, nasenartigen Fortsatz. Basis von Pereiopod V schlank, rechteckig; Basen der Pereiopoden VI und VII schlank, aber \pm rechtwinklig, schwach geschwungen, im ganzen wenig verbreitert. Pereionsegment IV deutlich verlängert. Urosomsegmente I bis III nicht verwachsen. Urosomsegment I mit dorsodistalem, gerundeten Fortsatz. Telson horizontal angeordnet, kleiner als Urosomsegment I, löffelartig durch Aufwölbung der Lateralränder.

Ty pus a r t: Thaumatelsonella kingelepha sp. $\mathrm{n}$.

$\mathrm{N}$ a m e : Nimmt Bezug auf generelle Übereinstimmung mit den charakteristischen Merkmalen der Thaumatelsoninen; Diminutivum telsonella bezieht sich auf das kleinflächige Telson.

Beziehungen: Eine äußerliche Gemeinsamkeit ist innerhalb der Thaumatelsoninen durch die Ausbildung eines dorsodistalen Fortsatzes sowohl an Schaftglied 1 der Antenne I als auch an Urosomsegment I mit den Gattungen Parathaumatelson, Pseudothaumatelson und Chucullba gegeben. Parathaumatelson und Chucullba sind vor allem abgegrenzt durch die gleich gestalteten Gnathopoden I und II, den fehlenden MandibelPalpus und die basal verwachsenen Urosomsegemente II und III; darüber hinaus sind die Laden der Maxille II reduziert und die Uropoden bei Lateralansicht gestaffelt angeordnet (Reichweite von Up I nach Up III abnehmend); die Reduzierung des Urosoms ist weiter fortgeschritten. Trotz wesentlicher äußerer Übereinstimmung ist Pseudothaumatelson deutlich durch den eingliedrigen Mandibel-Palpus getrennt. Bei Pseudothaumatelson erscheint die Größe der Urosomsegmente reduziert, der Dorsodistalfortsatz an Urosomsegment I ist jedoch deutlich länger als bei Thaumatelsonella.

Gegenüber der neuen Gattung weist Pseudothaumatelson mit dem eingliedrigen Mandibel-Palpus aber auch mit der Größenreduktion des Urosoms stärker abgeleitete Merkmale auf.

\section{Thaumatelsonella kingelepha sp. $\mathrm{n}$.}

(Abb. 1, J und $\mathrm{K}_{\mathrm{i}}$ Abb. 2, 3)

Holotypus: \&, 2,5 mm, ZMB; 22. Febr. 1986, M. Rauschert leg.

L oc us typicus: Antarktis, King George Island, Fildes Strait, Sublitorali Station: 30/49 A; Substrat: Schwämme und Bryozoen auf Hartböden; Fundort starken Gezeitenströmen ausgesetzt. Wassertiefe: $50 \mathrm{~m}$ (Gerät: Dredsche, 0,3 $\mathrm{mm}$ Maschenweite).

Pa ra t y p e $n$ : Antarktis, King George Island, Sublitoral; Fundort wie Holotypus. 1 ๙ , 2,1 mm und 2 \%, 2,4 und $2,6 \mathrm{~mm}$; ZMB. 1 o , 1,9 mm; ZMH: K 53480 .

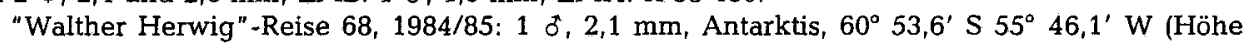
Elephant Island); "Walther Herwig"-Station 171, Hol 2; Wassertiefe $260 \mathrm{~m}$, Substrat: schlickiger Feinsand (Gerät: Greifer nach van Veen, 0,1 m²). ZMH K 33481 . Island.

Et ymologi e: Anagramm aus den bisherigen Fundorten King George Island und Elephant

Beschreibung: Kopf etwa 1,5fache Länge von Pereionsegment I, mit kurzem Rostrum. Kopfseitenlappen dreieckig vorspringend, spitz (oberer Rand nahezu gerade, unterer leicht konvex). Vordere Ventralecke winklig. A ugen groß. Pe reionsegmen t IV in beiden Geschlechtern erheblich verlängert (mehr als doppelte Länge von Segment III). Epimeralplatten 1 und 2 ventrodistal mit kleinem Zahn, -platte 3 ventrodistal gerundet ausgezogen $\left(60^{\circ}\right)$. 


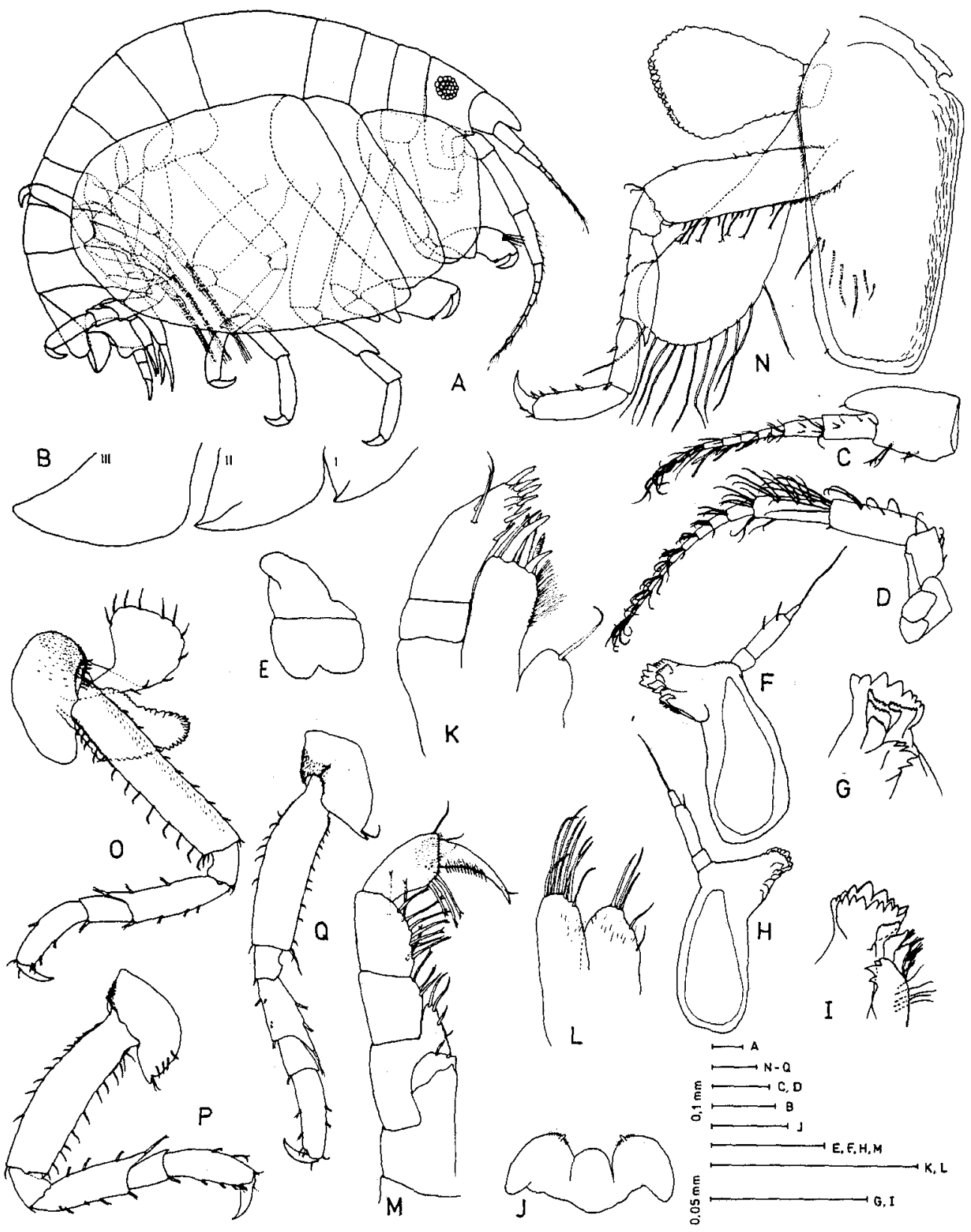

Abb. 2. Thaumatelsonella kingelepha gen. und sp. n.; A-F, J-Q: Holotypus, $q$. G und I: Paratypus, $\delta, 1,9 \mathrm{~mm}$. H und L: Paratypus, ơ, 2,1 mm. - A: Habitus (lateral). B: Epimeralplatten I-III. C: Antenne I. D: Antenne II. E: Oberlippe-Epistom-Komplex. F-I: Mandibel (rechte Md: F und G; linke Md: H und I). J: Unterlippe. K: Maxille I. L: Maxille II. M: Maxilliped. N-Q: Pereiopod III, V-VII 


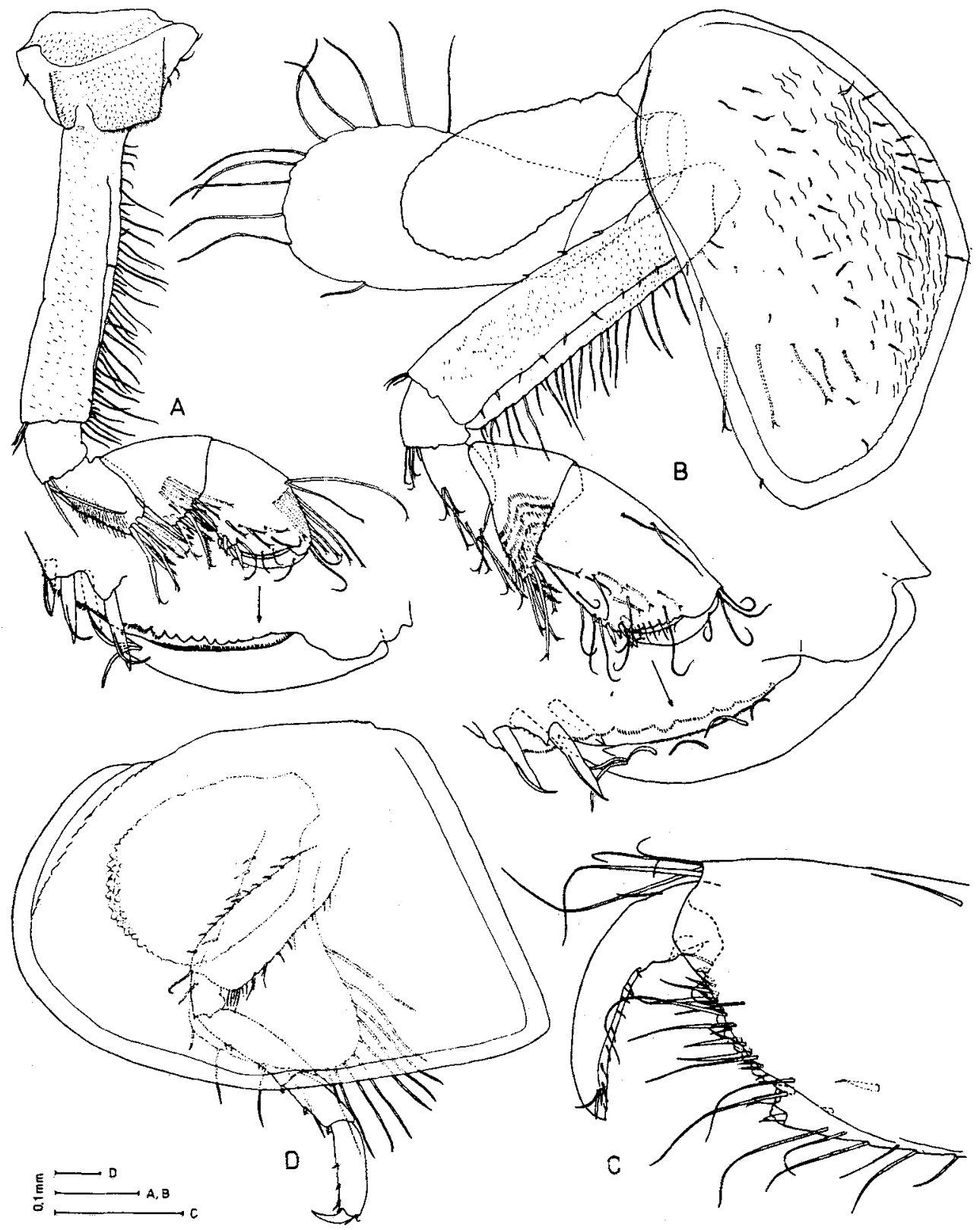

Abb. 3. Thaumatelsonella kingelepha gen. und sp. n. A, B und D: Holotypus, 9 . C: Paratypus, $\delta^{*}$ 2,1 mm. - A: Gnathopod I. B: Gnathopod II. C: Gnathopod II; Palma und Dactylus. D: Pereiopod IV 
U r o s o m s e g m e n t I mit dorsodistalem, gerundeten Fortsatz (Dorsalrand gerade verlaufend), Fortsatz übergreift proximale Hälfte des horizontal liegenden Telsons.

Antenne I kürzer als A II; Geißel erreicht etwa Ende von Stielglied 5 der A II. Stielglied $1 \mathrm{mit}$ dorsodistalem, nasenartigen, abgerundeten Fortsatz, der proximale Hälfte von Stielglied 2 übergreift. Stielglied 2 und 3 erreichen etwa $3 / 4$ bzw. 1/2-2/3 der Länge von Stielglied 1. Nebengeißel sehr kurz, eingliedrig, distal beborstet (1-3 Borsten). Geißel: bis zu 7 Gliedern, Endglied distal dicht beborstet; Aesthetasken vorhanden. Antenne II: Schaftglied 5 kürzer als -glied 4 (etwa 90\%). Geißel: bis zu 9 Gliedern; Geißelglied 1 länger oder kürzer als nächstfolgende; Geißelbewehrung: ausschließlich Borsten: E p is t o m gleichmäßig flach gewölbt. O b e r lip p e zweilappig, leicht asymmetrisch. Mandibe l: Pars incisiva gezähnt. Lacinia mobilis, links: breit, wie Hauptschneide gezähnt; rechts: deutlich schmaler und nur fein gezähnt (gekerbt). Stachelreihe: zahnartige, distal abgestutzte Stacheln (links 3, rechts bis zu 4). Molar: konische Protuberanz, gezähnt. Palpus 3gliedrig; Glied 2 am längsten, Glieder 1 und 3 erreichen $1 / 3-1 / 2$ der Länge von Glied 2; Beborstung: Glied 1 nackt, Glied 2 im distalen Abschnitt 1 oder 2 Borsten, Glied 3 distal 1 lange Endborste. Un t e r l i p p e : Innenlappen verwachsen; Hauptlappen an innerer Distalrundung mit kurzem, stiftförmigen Fortsatz (3zackig). Maxille I: Innenlade kurz zungenförmig, distal mit 1 Borste. Außenlade mit 6 z. T. gekämmten zahnartigen Stacheln (äußerer Stachel schlank, glatt; 2. Stachel von innen kurz). 2gliedriger Palpus überragt $\mathrm{AL}_{\text {; }}$ Glied 1 erreicht etwa $1 / 4$ der Länge von Glied 2, das distal und distomedial zahnartige Stacheln aufweist, unterhalb dieser Stacheln 1 oder 2 Borsten. Maxill e II : schlankere Außenlade überragt konisch geformte Innenlade; AL vorwiegend distal beborstet, Medialrand der IL mit 2 Borsten (beide LadenOberflächen mit verstreuter, feiner Behaarung). $\mathrm{Maxill}$ ipe d: Länge der Innenlade beträgt bis zu $4 / 5$ der Außenrandlänge des folgenden Gliedes; abgestutzter Distalrand nur mit 1 Borste und 1 zahnartigen Stachel, mediale Ecke leicht zahnartig ausgebildet; unterhalb des Distalrandes auf mundwärts gerichteter Fläche ein Absatz mit 1 kurzen Borste (medial angeordnet). Außenlade triangulär, etwa so lang wie breit, erreicht etwa die Hälfte des folgenden Gliedes, distal 1 oder 2 Borsten; Medialrand mit kleinen Zahnbildungen und 2 Stacheln. Palpus 4gliedrig, Glieder 1-3 etwa von gleicher Länge (Außenrand), Glied 3 distal verbreitert; Glied 4 klauenartig, Insertion teilweise von Glied 3 überragt; Innenrand mit feinen Kammstacheln (Oberfläche dorsal feiner bestachelt); Beborstung: Glied 1 an innerer Distalecke, Glied 2 auf medialer Oberfläche, Glied 3 am Distomedialrand (Borsten distal gegabelt).

Pereiopoden I und II als subchelate Gnathopoden ausgebildet; Gnp I deutlich kleiner als Gnp II. Lange Borsten der Bewehrung z. T. apikal gegabelt (neben kräftiger Hauptspitze eine fein auslaufende Spitze). Gn at hopod I : Coxalplatte stark reduziert, Basisansatz kaum überdeckend, subquadratisch (trapezförmig), mit Haarfilz besetzt, Ventralrand fast gerade. Basis im ganzen schlank; Vorderfläche durch laterale Leistenbildung mit Grube (Raum für angewinkelten Carpus sowie vorderen PropodusAbschnitt), innerer Basis-Vorderrand mit längeren Borsten besetzt. Merus durch ausgelappten gerundeten Hinterrand deutlich gegen Carpus abgesetzt; Hinterrand etwa so lang wie Carpus-Vorderrand; distaler Merus-Abschnitt mit kurzen, feinen Stacheln bewehrt; Auslappungsrand mit langen, glatten sowie kürzeren, im distalen Teil gefiederten Borsten besetzt. Carpus triangulär erreicht etwa $3 / 4-4 / 5$ der Propodus-Länge (Vorderrand), ausgelappt; Carpus-Beborstung: auf innerer Oberfäche eine schräge Borsten- 
reihe; Auslappung mit gefiederten Borsten (wie an Merus-Auslappung). Propodus \pm oval; distal zur Mitte des stark konvex verlaufenden Vorderrandes auf der inneren Oberfläche eine auffällige, den Rand stufenartig unterbrechende Borstenreihe; Hinterrand gerade verlaufend, gerundet in die nur geringfügig kürzere Palma übergehend; Übergangsbereich fein gezähnt, hier stehen 2 Paar (Typus, ๆ) oder 3 Palma-Grenzstacheln. Palma im ganzen schräg gestellt; Palmarand gerade bis schwach konvex verlaufend, lappig gekerbt bis gezähnt; Palmabereich mit einigen Borsten besetzt. Dactylus erreicht Palmarecke; Innenrand sehr fein gekämmt, in Höhe des Nagelansatzes mit Zahnbildung. Gn a thopod II : Gesamthöhe der Coxalplatte länger als Basis-Vorderrand; Vorderrand im ganzen stark ausgebuchtet, konvex (im Distalabschnitt nahezu gerade); Ventralrand gerade bis schwach konvex, Hinterrand gestreckt-sigmoid verlaufend. Basis lang rechteckig, distad etwas verbreitert; Vorderrand-Länge erreicht bis zu $90 \%$ der Gesamtlänge von Carpus und Propodus (Vorderrand); Leistenbildung wie an Gnp I. Merus ausgezogen (an der Spitze mit Borsten, beim $q$ reichere Beborstung), Hinterrand etwa so lang wie Carpus-Vorderrand (beim to etwas länger). Carpus erreicht $45 \%\left(\delta^{*}\right)$ bis $>55 \%(\$)$ der Propodus-Länge (Vorderränder), ausgelappt; Vorderrand der Auslappung erscheint durch Borstenansätze leicht gestuft. Propodus im ganzen rechteckig, Vorder- und Hinterrand leicht konvex; Breite erreicht 55-60\% der Länge; Hinterrand länger als Palma, sehr fein gekörnt. Palma schräg gestellt; Palmarecke beim $\delta$ mit 3 Stacheln und einer deutlichen Stufe (bei adulten $\delta$ ) markiert (Stufenrand gekerbt); beim $q$ dagegen durch 2 Paar Stacheln (Stufe nur undeutlich ausgebildet, Rand sehr fein gekämmt) gekennzeichnet; die Palma selbst ist lappig gewellt (Lappenränder beim $\uparrow$ sehr fein gekämmt; beim $\delta$ ist die Lappung stärker ausgeprägt, Ränder aber nackt) und zeigt geraden bis leicht konvexen Verlauf; Palmabereich mit Stacheln und Borsten bewehrt. Beim $q$ erreicht der Dactylus nicht die Palmarecke (nur bis zum inneren Stachelpaar); Innenrand submarginal mit Borsten, Rand selbst beim ơ glatt, beim $q$ fein gekämmt; in Höhe des Nagelansatzes mit kleinem Zahn.

Perei o poden III und IV von gleicher Gestalt, schlank. Basis an P III etwas länger. Merus-Vorderrand etwas ausgezogen. Dactylus etwa $1 / 3-1 / 2$ der Propodus-Länge. Coxalplatte III rechteckig; distal schmaler; Gesamtlänge deutlich größer als die der Basis, ventrodistale Ecken gerundet. Coxalplatte IV schildförmig vergrößert, deutlich länger als hoch (Höhe etwa $2 / 3-3 / 4$ der Länge); Vorderrand etwas cranioventrad vorgezogen, aber gerade verlaufend, Ventralecke gerundet; Ventralrand allmählich in den stark konvexen Hinterrand übergehend.

Pereiopoden V-VII etwa von gleicher Gestalt, schlank, Bewehrung gering ausgebildet. Coxalplatten länger als hoch, caudal ausgelappt; caudaler Abschnitt an P V gerundet (Distalrand kann Einkerbung mit kleiner Borste aufweisen), an P VI-VII ventrodistad mit Zahnbildung (oberhalb des Zahnes 1 Borste). Basen schlank, rechteckig, an P VI und VII im ganzen schwach verbreitert und leicht geschwungen (Hinterränder leicht konkav, Vorderränder konvex). Dactylus kräftig, erreicht bis zu $50 \%$ der jeweiligen Propodus-Länge. P e r e i o p od V: Basis linear, so lang wie Glieder 4-6 zusammen genommen. Merus-Hinterrand etwas ausgezogen. P e r e i o pod VI: Gesamtlänge der Glieder 4-6 etwas länger als Basis. Spitzer Auszug des Merus-Hinterrandes übergreift etwa die proximale Carpus-Hälfte. Pe re i o p o d VII: Gestalt entspricht im ganzen der von P VI, jedoch erreicht Basis nicht die Gesamtlänge der Glieder 4-6, und der Merus ist kürzer. Spitzer Auszug des Merus-Hinterrandes übergreift etwa 2/3 der Carpus-Länge. 
Ki e men: sackförmig, an P II-VI (an P VI klein); bei $q$ ist die Kiemenoberfläche vor allem an P III-V durch kleine, zottige Ausstülpungen vergrößert. O o s t e g i t e zungenbis blattförmig, an P II-V. Ple op oden: normal. Uropoden I und III ragen etwa gleich weit nach hinten, Up II kürzer. Äste von Up I und II lanzettförmig, stachellos, Ränder sehr fein gekämmt, Schäfte dagegen bestachelt. U r o p od I: Schaft länger als Außenast; Außenast geringfügig länger als Innenast. U ropo d II : Schaft etwa so lang wie Innenast; Innenast länger als Außenast. Uropod III einästig; Ast 2gliedrig, Schaft- und Astglieder nahezu von gleicher Länge. Bestachelung des Schaft- sowie Basalgliedes am dorsad gerichteten Distalrand, 1 kleiner Stachel distoventral an Astglied 1. Tels on horizontal angeordnet, länger als breit (etwa 1:0,8), löffelartig durch aufgewölbte Lateralränder, marginal versteift, distal zugespitzt; nur marginale Beborstung in etwa $1 / 3$ ( 1 kurze Borste) und in $2 / 3$ der Länge ( 1 lange Borste gefolgt von 1 kurzen).

Färbung der lebenden Tiere: Augen rot; Körper gelblich, mit weißen Flecken; Pereionsegment IV dorsolateral mit rötlichem Fleck; Pereionsegment VII dorsal rötlich.

Verbreitung und Vorkommen: Antarktis, Shetland Islands (King George Island, Elephant Island). Tiefenbereich: 50 bis $260 \mathrm{~m}$.

Danksagungen. Das Material entstammt teilweise Benthosaufsammlungen, die im Rahmen des Projektes von Prof. Di. G. Hartmann (Universität Hamburg) ANTARKTISCHE POLYCHAETEN UND CRUSTACEEN durchgeführt wurden; dieses Projekt wurde von der Deutschen Forschungsgemeinschaft (DFG) unterstützt. Die Einbringung und Vorsortierung der Proben besorgte Dr. U. Mühlenhardt-Siegel (Zool. Institut und Zool. Museum, Hamburg). Für die Arbeiten im Gebiet der King George Island diente als Basis die sowjetische Antarktis-Station Bellingshausen. Die taxonomisch-systematische Bearbeitung der antarktischen Stenothoidae durch die Autoren wurde wesentlich gefördert durch gegenseitige Arbeitsaufenthalte, die vom jeweiligen gastgebenden Institut finanziert wurden. Dr. L. Sandberg (Naturhistoriska Riksmuseét, Stockholm) hat bereitwillig Typmaterial zur Verfügung gestellt.

\section{LITERATUR}

Barnard, J. L., 1972a. Gammaridean Amphipoda of Australia, Part I. - Smithson. Contr. Zool. 103, $1-333$.

Barnard, J. L., 1972b. The marine fauna of New Zealand: Algae-living littoral Gammaridea (Crustacea Amphipoda). - Mem. N.Z. oceanogr. Inst. 62, 1-216.

Barnard, J. L., 1974. Gammaridea Amphipoda of Australia, Part II. - Smithson. Contr. Zool. 139, 1-148.

Bellan-Santini, D. \& Ledoyer, M., 1986. Gammariens (Crustacea, Amphipoda) des lles Marion et Prince Edward. Campagne MD 08 du MS "Marion Dufresne" en 1976. - Boll. Mus. civ. Stor. nat. Verona 13, 349-435.

Gurjanova, E., 1938. Amphipoda Gammaridea of Siaukhu Bay and Sudzukhe Bay (Japan Sea). Rep. Japan Sea Hydrobiol. Exped. 1934 1, 241-404. (In Russ.).

Nicholls, G. E., 1938. Amphipoda Gammaridea. - Scient. Rep. Australas. Antarct. Exped. (Ser. C) $2(4), 1 \sim 145$.

Schellenberg, A., 1931. Gammariden und Caprelliden des Magellangebietes, Südgeorgiens und der Westantarktis. - Further zool. Results Swed. Antarct. Exped. 2 (6), 1-290.

Stebbing, T. R. R., 1888. Report on the amphipods collected by H. M. S. "Challenger" during the years 1873-1876. - Rep. scient. Results Voyage H. M. S. Challenger 29, 1-1737.

Walker, A. O., 1906. Preliminary descriptions of new species of Amphipoda from the "Discovery" Antarctic Expedition, 1902-1904. - Ann. Mag. nat. Hist. (Ser. 7) 18, 13-18. 